\title{
Biosensing using aligned-gap multiple split ring resonator at microwave frequency for possible application in halal industry
}

\begin{abstract}
A high-quality factor of split ring resonator (SRR) is an ideal structure for the development of compact and high sensitivity sensor. A microwave resonant method using aligned-gap rectangular SRR is presented in this work for measuring permittivity of dielectric materials. The sensing approach is based on detecting a shift in resonance frequency of the sensor when a dielectric material interacts with the electric field distribution between the gaps. The proposed metamaterial-based sensor was designed and fabricated on a Rogers RT5880 substrate having a dielectric constant of 2.2 and a thickness of $0.787 \mathrm{~mm}$ with a final dimension measured at $35 \times 14 \mathrm{~mm} 2$. Measured result shows good agreement with simulated ones as well as exhibiting high Q-factor to be used in sensing application. An obvious shift of resonance frequency is observed upon introduction of several solid samples with different dielectric value. The findings show possible application to halal sector.
\end{abstract}

Keyword: Dielectric sensing; Metamaterial; Permittivity; Resonant method; Split ring resonator 